\title{
Securing Low-cost RFID Systems: an Unconditionally Secure Approach
}

\author{
Basel Alomair ${ }^{\mathrm{a}}$, Loukas Lazos ${ }^{\mathrm{b}}$, and Radha Poovendran ${ }^{\mathrm{a}}$ \\ ${ }^{a}$ Network Security Lab (NSL), University of Washington, Seattle, USA \\ ${ }^{\mathrm{b}}$ Electrical and Computer Engineering Dept., University of Arizona, Tucson, USA \\ e-mail: \{alomair,rp3\}@uw.edu and llazos@ece.arizona.edu
}

\begin{abstract}
In this paper, we explore a new direction towards solving the identity authentication problem in RFID systems. We break the RFID authentication process into two main problems: message authentication and random number generation. For parties equipped with a good source of randomness and a secure cryptographic primitive to authenticate messages, the literature of cryptography is rich with wellstudied solutions for secure identity authentication. However, the two operations, random number generation and message authentication, can be expensive for lowcost RFID tags. In this paper, we lay down the foundations of a new direction towards solving these problems in RFID systems. We propose an unconditionally secure direction for authenticating RFID systems. We use the fact that RFID readers are computationally powerful devices to design a protocol that allows RFID readers to deliver random numbers to RFID tags in an unconditionally secure manner. Then, by taking advantage of the information-theoretic security of the transmitted messages, we develop a novel unconditionally secure message authentication code that is computed with a single multiplication operation. The goal of this work is to bring more research to the design of such unconditionally secure protocols, as opposed to the computationally secure protocols that have been proposed extensively, for the purpose of suiting the stringent computational capabilities of low-cost devices.
\end{abstract}

Keywords. RFID, unconditional security, authentication, secrecy

\section{Introduction}

While mutual authentication is a well-studied problem in the cryptographic literature, it becomes more challenging with the use of low-cost devices. Low-cost RFID tags, in particular, have limited computational capabilities that render them unable to perform sophisticated cryptographic operations. Hoping Moore's law will eventually render RFID tags computationally powerful, it might be tempting to consider the computational limitations of low-cost tags a temporary problem. The cost of tags, however, will remain a determining factor in the deployment of RFID systems in real life applications. When RFID technology is to replace barcodes to identify individual items, RFID tags will substantially contribute to the cost of these products. Even when the price of tags that can implement provably secure cryptography can be driven to 10 cents or less, it would still be impractical to attach them to low-cost items, e.g., 50-cent or cheaper products. When retailers are to choose between tags that can perform sophisticated cryptographic operations and cheaper tags that cannot, it seems inevitable that the cheaper tags will prevail. 
The problem of mutual authentication in RFID systems has been studied under different constraints. Juels and Pappu proposed the use of a public key cryptosystem to solve the problem of consumer privacy in RFID banknotes [1]. Golle et al. [2] proposed the universal re-encryption, where re-encryption of the ciphertext does not require the knowledge of the corresponding public key. Burmester et al. and Liang et al. proposed the use of one-way trapdoor functions in $[3,4]$ to solve the traceability problem in RFID system. As public key proposals might be suitable for some applications (e.g., banknotes [1], ePassports [5], credit cards [6], etc.), they are impractical for low-cost RFID tags.

Consequently, proposals based on the hardness of breaking symmetric key primitives have been the most popular solution for RFID systems. Such solutions include the use of symmetric key encryption [7,8,9], pseudorandom functions (PRF) [10,11,12], cryptographic hash functions $[13,14,15,16,17]$, or other NP-hard problems such as the linear parity with noise problem $[18,19,20,21,22]$. Although computationally secure symmetric key solutions are usually less expensive than asymmetric ones, they still require expensive operations and/or carefully designed iterations of complicated operations.

In order to come up with cheaper solutions, lightweight protocols that are not based on computational assumptions and require tags to perform only simple bitwise operations have been proposed, e.g., in [23,24,25]. Such proposals, however, were not based on rigorous security analysis and have been shown to have severe security flaws, see e.g., $[26,27,28]$ for analysis of specific protocols. In fact, it has been shown in [29] that simple bitwise operations cannot lead to secure authentication protocols.

As can be observed from the above examples, previous secure RFID protocols are all computationally secure. Hoping to meet the limited computational capabilities of lowcost tags, we start the search for unconditionally secure protocols for RFIDs. Unconditional security relies on the freshness of the keys rather than the hardness of solving mathematical problems. Thus, an appropriately designed unconditionally secure protocol will normally require less computational effort.

CONTRIBUTIONS. We propose the first UnConditionally Secure mutual authentication protocol for RFID systems (UCS-RFID). To minimize the computational effort on tags, we develop an unconditionally secure method for delivering random numbers from RFID readers to tags. Thus, allowing tags to benefit from the functionalities of random numbers without the hardware to generate them. Then, we take advantage of the secrecy of exchanged messages to develop a novel unconditionally secure technique for message authentication using only a single multiplication operation.

Organization. The rest of the paper is organized as follows. In Section 2, we give a brief background and discuss some related work. In Section 3, we state our model assumptions. In Section 4, we describe our UCS-RFID protocol. In Section 5, we give detailed security analysis of mutual authentication in the proposed UCS-RFID. In Section 6 , we extend our security model and formally address desynchronization attacks on the proposed system. In Section 8 we discuss tags' privacy in the proposed system. We conclude the paper in Section 9.

\section{Background and Related Work}

A typical RFID system consists of three main components: RFID tags, RFID readers, and a database. The RFID tags, or transponders, are small devices with minimal storage and 


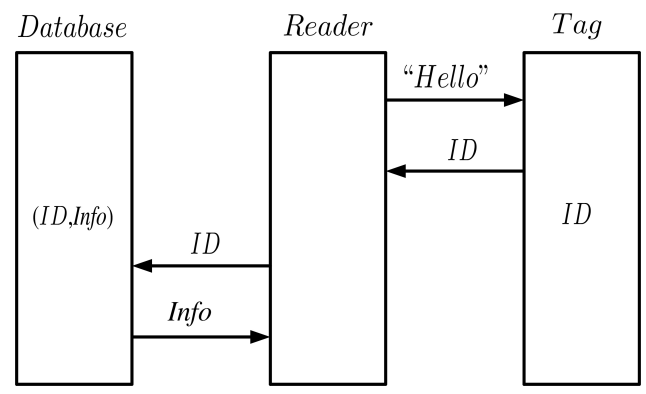

Figure 1. A simple identification protocol.

computational capabilities, equipped with a small antenna. Tags can be attached to an item and store information, such as a unique product $I D$, type of product, serial number, manufacturing date, expiration date, etc.

To retrieve information from an RFID tag, the tag must be interrogated by an RFID reader located within communication range of the tag. When an RFID reader interrogates a tag, the tag responds with a quantity that can be used by the reader to uniquely identify it. Figure 1 shows a simple protocol for the tag identification. The RFID reader broadcasts a "Hello" message to announce its presence to any tag within its communication range and charge the tags if they are passive. The RFID tag responds to the reader's request by transmitting its unique $I D$. The tag's unique $I D$ is then used by the reader to lookup the database for information related to the item carrying the RFID tag.

Transmitting the unique $I D$ of an RFID tag in clear text, as in the simple identification protocol in Figure 1, can lead to variety of attacks. An adversary observing one identification run can easily impersonate the tag by emitting its unique $I D$ to identify itself. An active adversary can modify the tag's $I D$ before it reaches the reader, leading the reader to possibly identify another tag. Furthermore, a tag can be traced by its unique $I D$, thus compromising the privacy of the user carrying the tag.

To mitigate the vulnerabilities of the simple identification protocol, the problem of mutual authentication in RFID systems has been studied under different constraints. Juels and Pappu have suggested the use of a public key cryptosystem to solve the problem of consumer privacy in RFID banknotes [1]. Avoine pointed out possible limitations on the security of their protocol in [30]. Golle et al. proposed the universal re-encryption, where re-encryption of the ciphertext does not require the knowledge of the corresponding public key [2]. Burmester et al. proposed the use of one-way trapdoor functions to address privacy in RFID systems [3]. Liang et al. analyzed the protocol of [3] showing that it can reveal secret information, and proposed security improvements [4].

Symmetric key solutions, however, have appeared more frequently than public key ones. Feldhofer et al. proposed the use of a 128-bit version of the Advanced Encryption Standard (AES) [7,8]. Their implementation requires 3628 gates in expense of more clock cycles. One-way cryptographic hash functions have been used extensively in the design of authentication protocols for RFID systems (see, e.g., [31,32,33,34]). Privacy and security issues of RFID systems based on one-way hash functions have also been studied extensively (see, e.g., $[13,35,15,36])$.

Another symmetric key solution that has been studied is based on the human-tocomputer authentication protocol designed by Hopper and Blum in [37]. Juels and Weis proposed $\mathrm{HB}^{+}$, a lightweight authentication protocol based on the Learning Parity with 
Noise (LPN) problem [18]. Gilbert et al. showed a successful linear time active attack on the $\mathrm{HB}^{+}$protocol in [27], and proposed an improvement to the security and efficiency of $\mathrm{HB}$ in [19]. Katz and Smith analyzed the $\mathrm{HB}$ and $\mathrm{HB}^{+}$in the large error case [38].

Protocols that require only bitwise operations were proposed in [23,24,25,39]. In [23], Vajda and Buttyan proposed lightweight cryptographic primitives for tag authentication based on bitwise operations. In [40], Defend et al. described security vulnerabilities in Vajda and Buttyan protocols. In [24,25,39], Peris-Lopez et al. proposed LMAP, $\mathrm{M}^{2} \mathrm{AP}$, and EMAP, mutual authentication protocols that require the tag to do simple bitwise operations and modulo additions. These protocols, however, have been analyzed and shown to be vulnerable to attacks. In [41], Li and Wang describe active attacks against LMAP and $\mathrm{M}^{2} \mathrm{AP}$ that require $O(N)$ interactions between the adversary and the tag to extract its $I D$ of length $N$. In [26], Li and Deng described active attacks against EMAP that lead to revealing the tag's $I D$. In [28], Alomair et al. showed how the $I D$ of a tag using the $\mathrm{M}^{2} \mathrm{AP}$ or the EMAP protocols for authentication can be extracted by passively observing $O(\log N)$ interactions between the tag and an authorized reader.

To formalize security analysis in RFID systems, Avoine proposed an adversarial model suitable for RFID systems and analyzed several well-known RFID protocols [42]. Ha et al. proposed a new formal model for analyzing location privacy in RFID system [43]. Ma et al. derived the relations between different notions of privacy in RFID systems and formalized the necessary and sufficient conditions to achieve privacy in RFID systems [12]. Li and Ding addressed the security requirements for RFID based supply chains [44].

Survey papers on the security and privacy of RFID systems include, but are not limited to, $[45,46,47,48]$. An up-to-date report listing known broken RFID protocols with detailed descriptions of the suggested attacks is maintained by Van Deursen and Radomirovic [49].

\section{System Model}

In this section, we describe our system model, adversarial model, security model, and preliminaries that will be used throughout the rest of the paper.

\subsection{Computational Capabilities}

We assume low-cost RFID tags identified via unique identifiers. Tags are assumed to be capable of performing bitwise (XOR) operations, in addition to modular multiplication and addition. RFID tags are not assumed to have the capability of performing traditional computationally-secure cryptographic primitives such as MACs, hash functions, random numbers generations, etc.

RFID readers are powerful devices capable of performing sophisticated cryptographic primitives. Readers are also assumed to be connected to the database via a secure link (whether by establishing a secure wireless channel or using wired connection) in order to retrieve information about tags. Addressing the security between RFID readers and the database is beyond the scope of this paper. 


\subsection{Adversarial Model}

We assume an adversary with a complete control over the communication channel. The adversary can observe messages exchanged between readers and tags, initiate communication with a reader or a tag, modify messages exchanged between the authorized reader and tags in the system, block messages and replayed them later, and generate messages of her own. We do not consider an adversary whose only goal is to jam the communication channel.

The adversary is modeled as a polynomial-time algorithm. Similar to the adversarial model proposed by Avoine in [42], given a tag $T$ and a reader $R$, we assume that the adversary has access to the following oracles:

- $\operatorname{Send}\left(R, m_{1}, x_{2}, m_{3}\right)$ : The adversary executes the protocol, acting as a tag. The adversary sends $m_{1}$ to identify itself to $R$; receives the reader's response, $x_{2}$; and authenticate itself with $m_{3}\left(x_{2}\right)$. This oracle models the adversary's ability to impersonate a tag in the system.

- $\operatorname{Query}\left(T, x_{1}, m_{2}, x_{3}\right)$ : The adversary acts as the reader in an instance of the protocol. The adversary interrogates $T$; receives the tag's response, $x_{1}$; sends the message $m_{2}\left(x_{1}\right)$ to authenticate itself; and receives $x_{3}$. This oracle models the adversary's ability to impersonate valid readers.

- Execute $(T, R)$ : The tag $T$ and the reader $R$ execute an instance of the protocol. The adversary eavesdrops on the channel; this oracle models the adversary's ability to monitor the channel between tag and reader.

Observe that in practical RFID systems, unlike the Send and Query oracles, the adversary does not have complete control over the number of Execute oracles she can call on a particular tag. This is due to the fact that the Execute oracle simulates a complete protocol run between authorized reader-tag pairs. Thus, unless the adversary is physically capturing a tag, she does not have a complete control of when the tag is in the vicinity of an authorized reader.

\subsection{Security Model}

As in [50], we define honest protocol runs as follows: A mutual authentication protocol run is said to be honest if the parties involved in the protocol run use their shared key to exchange messages, and the messages exchanged in the protocol run have been relayed faithfully (without modification).

Another term that will be used in the reminder of the paper is the definition of negligible functions.

Definition 1 (Negligible Function [51]) A function $\gamma: \mathbb{N} \rightarrow \mathbb{R}$ is said to be negligible if for any nonzero polynomial $p$, there exists $N_{0}$ such that for all $N>N_{0},|\gamma(N)|<$ $1 /|p(N)|$. That is, the function is said to be negligible if it converges to zero faster than the reciprocal of any polynomial function.

We now provide a formal definition of secure mutual authentication for RFID systems.

Definition 2 (Secure Mutual Authentication [50]) A mutual authentication protocol for RFID systems is said to be secure if it satisfies the following conditions: 
1. No information about the secret keys of the RFID tag is revealed by observing messages exchanged in protocol runs.

2. Honest protocol $\Rightarrow$ Authentication: if the protocol run is honest, the tag-reader pair must authenticate each other with probability one.

3. Authentication $\Rightarrow$ Honest protocol: the probability of authentication when the protocol is not honest is negligible in the security parameter.

To model the adversary's attempt to authenticate herself to a reader (tag), we propose the following game between the challenger $\mathcal{C}$ (the RFID system) and an adversary $\mathcal{A}$.

1. $\mathcal{A}$ signals $\mathcal{C}$ to begin the game.

2. $\mathcal{C}$ chooses a tag, $T$, at random, and a reader, $R$, and gives them to $\mathcal{A}$.

3. $\mathcal{A}$ calls the oracles Query, Send, and Execute using $T$ and $R$ (this is the data collecting phase).

4. $\mathcal{A}$ decides to stop and signals $\mathcal{C}$ to move on to the next phase.

5. $\mathcal{A}$ Send (Query) $\mathcal{C}$ as if it is a tag (reader) in the system (this is the actual attempt to break the security of the system).

6. If $\mathcal{A}$ is authenticated as a valid tag (reader), $\mathcal{A}$ wins the game.

Definition 2 implies that the protocol achieves secure mutual authentication only if the adversary's probability of winning the game is negligible in the security parameter.

To analyze privacy of the proposed protocol, the following definition gives two degrees of privacy in RFID systems.

Definition 3 For RFID tags, we define two notions of privacy as follows.

1. Passively Private: a tag is said to be passively private if two instances of the tag identifiers, separated by a successful mutual authentication with an authorized reader, cannot be correlated by unauthorized observers.

2. Actively Private: a tag is said to be actively private if two instances of the same tag cannot be correlated by unauthorized observers, even if the tag does not achieve mutual authentication with an authorized reader in between.

As can be inferred by their names, the first notion implies privacy against passive adversaries only, while the second notion implies privacy against both passive and active adversaries. To see this, observe that the definition of actively private tags requires that tags cannot be traceable by their responses even if tags do not achieve mutual authentication with valid readers. This is to model the adversary's ability to impersonate valid readers by actively interrogating tags and observing their responses. The definition of passively private, on the other hand, emphasizes that tags cannot be traceable before and after their mutual authentication with a valid reader. This models adversary's who are passively monitoring the communication between authorized reader-tag pairs.

The privacy of a tag can be quantified using the advantage of an adversary $\mathcal{A}$ of identifying a tag $T_{a}$ in the presence of another tag $T_{b}$, defined as follows [42]:

$$
\operatorname{Adv}_{\mathcal{A}}=2\left(\operatorname{Pr}\left(T_{g}=T_{a}\right)-0.5\right)
$$

where $T_{g}$ denotes the adversary's guess, and $\operatorname{Pr}\left(T_{g}=T_{a}\right)$ denotes the probability that the adversary picks $T_{a}$ over $T_{b}$. For $\operatorname{Pr}\left(T_{g}=T_{a}\right)=\frac{1}{2}$, the adversary's decision is based 
on a random guess and, hence, no advantage is gained by observing exchanged messages $\left(A d v_{\mathcal{A}}=0\right)$. For $\mathrm{Adv}_{\mathcal{A}}=0$, the tag is considered to be untraceable.

\subsection{Preliminaries}

The following notations will be adopted throughout the paper. For a finite integer $p, \mathbb{Z}_{p}$ will denote the finite integer ring with the usual addition and multiplication modulo $p$. $\mathbb{Z}_{p}^{*}$ will denote the multiplicative group modulo $p$; that is, the subset of $\mathbb{Z}_{p}$ with elements relatively prime to $p$. For the special case at which $p$ is a prime integer, $\mathbb{Z}_{p}^{*}$ will contain all non-zero elements of $\mathbb{Z}_{p}$; that is, $\mathbb{Z}_{p}^{*}=\mathbb{Z}_{p} \backslash\{0\}$. $\mathbb{Z}_{p}^{*}$ and $\mathbb{Z}_{p} \backslash\{0\}$ will be used interchangeably to emphasize the multiplicative property or the exclusion of the zero element, respectively. Throughout the rest of the paper, random variables will be represented by bold font symbols, whereas the corresponding non-bold font symbols represent specific values that can be taken by these random variables.

The following are two important properties of the integer ring $\mathbb{Z}_{p}$ that will be used in the security analysis of UCS-RFID.

Lemma 1 For any two integers $\alpha$ and $\beta$ in $\mathbb{Z}_{p}$, if $p$ is a prime integer and $p$ divides $\alpha \beta$, then one of the integers $\alpha$ and $\beta$ must be the zero element in $\mathbb{Z}_{p}$. Formally, if $p$ is a prime integer, $\{\alpha \beta \equiv 0 \bmod p\}$ implies that $\{\alpha \equiv 0$ OR $\beta \equiv 0 \bmod p\}$.

Lemma 2 Let $p$ be a prime integer. Then, given an integer $k \in \mathbb{Z}_{p}^{*}$, for an $r$ uniformly distributed over $\mathbb{Z}_{p}$, the value $\delta \equiv r k \bmod p$ is uniformly distributed over $\mathbb{Z}_{p}$.

Lemma 1 states that, for a prime integer $p$, the ring $\mathbb{Z}_{p}$ is an integral domain. Lemma 2 is a direct consequence of the fact that, for a prime integer $p$, the ring $\mathbb{Z}_{p}$ is a field. One more definition that is vital for this paper is the definition of Shannon's perfect secrecy [52].

Definition 4 (Perfect Secrecy [53]) For a plaintext $m$ and its corresponding ciphertext $\varphi$, the cipher is said to achieve perfect secrecy if $\operatorname{Pr}(\boldsymbol{m}=m \mid \boldsymbol{\varphi}=\varphi)=\operatorname{Pr}(\boldsymbol{m}=m)$ for all plaintext $m$ and all ciphertext $\varphi$. That is, the a posteriori probability that the plaintext is $m$, given that the ciphertext $\varphi$ is observed, is identical to the a priori probability that the plaintext is $m$.

\section{The proposed UCS-RFID System}

Based on pre-defined security requirements, a security parameter, $N$, is specified and a $2 N$-bit prime integer, $p$, is chosen. Initially, each tag is loaded with an $N$ bit long identifier, $A^{(0)}$, and a secret key composed of five subkeys, i.e., $K^{(0)}=$ $\left(k_{a}^{(0)}, k_{b}^{(0)}, k_{c}^{(0)}, k_{d}^{(0)}, k_{u}^{(0)}\right)$. The length of $k_{a}$ and $k_{d}$ is $N$ bits, while $k_{b}, k_{c}$, and $k_{u}$ are $2 N$-bit long. The subkeys, $k_{a}^{(0)}$ and $k_{d}^{(0)}$, and the identifier, $A^{(0)}$, are drawn independently and uniformly from $\mathbb{Z}_{2^{N}} ; k_{b}^{(0)}$ is drawn uniformly from $\mathbb{Z}_{p}$; while $k_{c}^{(0)}$ and $k_{u}^{(0)}$ are drawn independently and uniformly from $\mathbb{Z}_{p}^{*}$. The subkeys $k_{a}, k_{b}, k_{c}$, and $k_{d}$ will be used to generate messages exchanged in protocol runs, while the sole purpose of $k_{u}$ is for updating the secret keys to maintain certain properties (details are discussed later). 


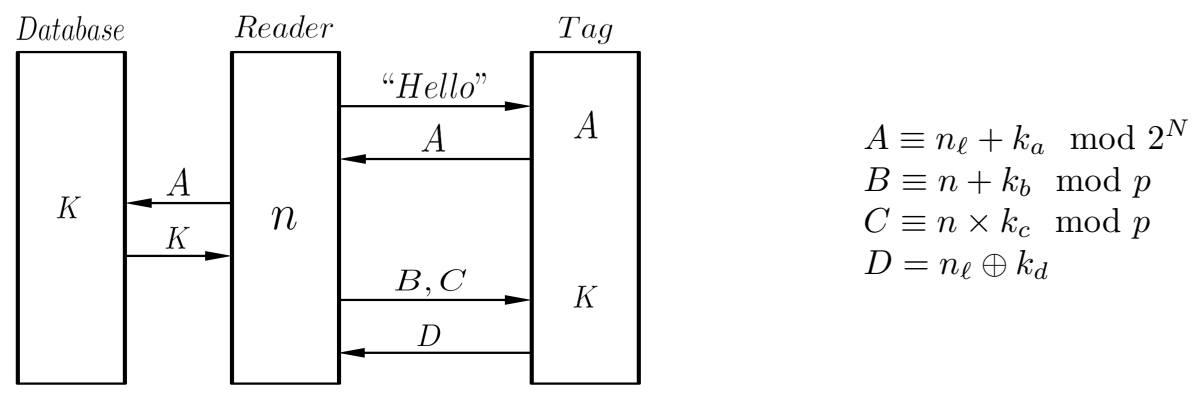

Figure 2. A schematic of one instance of the protocol.

The security of the protocol relies on the reader's ability to convey a random nonce to the tag in an authenticated and secret manner (inspired by the information theoretically secure authenticated encryption proposed in [54]). When an RFID reader interrogates a tag within its communication range, the tag responds with its identifier, $A$. Once the tag has been identified, the reader generates a $2 N$-bit long random nonce, $n$, and delivers it to the tag. If the reader is authenticated successfully, the received $n$ will be used by the tag to authenticate itself to the valid reader.

For the rest of the paper, quantities involved in the generation of exchanged messages in different protocol runs will be differentiated by superscripts. When differentiation between protocol runs is unnecessary, superscripts will be dropped for ease of notation.The proposed UCS-RFID enables the mutual authentication between an RFID reader and a tag by executing four phases: a tag identification phase, a reader authentication phase, a tag authentication phase, and a key updating phase. Figure 2 depicts a single protocol run of the proposed UCS-RFID.

\subsection{Tag Identification Phase}

In order to carry out the authentication process, the reader must identify the tag it is communicating with to access its key information.

Step 1. The reader announces its presence by broadcasting a "Hello" message.

Step 2. The tag responds to the "Hello" message by sending its current identifier, $A$.

Step 3. The reader looks up the database for the key $K=\left(k_{a}, k_{b}, k_{c}, k_{d}, k_{u}\right)$ corresponding to the tag's current identifier, $A .{ }^{1}$ If $A$ is not recognized as a valid identifier, the tag is rejected.

\subsection{Reader Authentication Phase}

This is one of the most important phases in the proposed protocol. In this phase, the RFID reader authenticates itself to the tag by proving its knowledge of the tag's subkeys

${ }^{1}$ Database management is out of the scope of this work. 
$k_{b}$ and $k_{c}$. More importantly, the reader delivers a nonce, $n$, to the tag in an authenticated and perfectly secret manner.

Step 4. The reader generates a $2 N$-bit random nonce, $n$, drawn uniformly from the multiplicative group $\mathbb{Z}_{p}^{*}$. We emphasize that $n$ must be an unpredictable nonce; predictable nonces such as time stamps do not induce the required randomness.

Step 5. With $k_{b}, k_{c}$, and $n$, the reader broadcasts two messages, $B$ and $C$, generated according to the following formulas:

$$
\begin{array}{ll}
B \equiv n+k_{b} & \bmod p \\
C \equiv n \times k_{c} & \bmod p .
\end{array}
$$

Step 6. Upon receiving $B$ and $C$, the tag extracts $n$ from message $B$ and verifies its integrity using message $C$. The reader is authenticated if and only if the following integrity check is satisfied,

$$
\left(B-k_{b}\right) \times k_{c} \equiv C \bmod p .
$$

If the integrity check of equation (4) does not pass, the reader will not be authenticated and the tag will abort the protocol.

\subsection{Tag Authentication Phase}

In the tag authentication phase, the tag is authenticated by its ability to extract the correct nonce, $n$, and its knowledge of the secret key $k_{d}$.

Step 7. If the reader failed the authentication process, the tag aborts the protocol. Otherwise, the tag broadcasts message $D$, given by

$$
D=n_{\ell} \oplus k_{d}
$$

where $n_{\ell}$ denotes the $N$ most significant bits of $n$.

Step 8. Upon receiving $D$, the reader authenticates the tag by verifying that the received $D$ is equal to $n_{\ell} \oplus k_{d}$. Otherwise, the tag is rejected.

\subsection{Key Update Phase}

After a mutual authentication between the RFID reader and the tag is achieved, the parameters are updated at the database and the tag for the next mutual authentication run.

Step 9. The reader and the tag update the key, $K$, and the tag identifier, $A$. Let $A^{(m)}$, $k_{i}^{(m)}$, and $n^{(m)}$ denote the identifier $A, k_{i}$, and $n$ used to execute the $m^{t h}$ protocol run; let $n_{r}$ denotes the $N$ least significant bits of $n$. Then, the parameters are updated as follows, 


$$
\begin{aligned}
& k_{a}^{(m+1)}=n_{r}^{(m)} \oplus k_{a}^{(m)}, \\
& k_{b}^{(m+1)} \equiv k_{u}^{(m)}+\left(n^{(m)} \oplus k_{b}^{(m)}\right) \bmod p, \\
& k_{c}^{(m+1)} \equiv k_{u}^{(m)} \times\left(n^{(m)} \oplus k_{c}^{(m)}\right) \bmod p, \\
& k_{d}^{(m+1)}=n_{r}^{(m)} \oplus k_{d}^{(m)}, \\
& k_{u}^{(m+1)} \equiv k_{u}^{(m)} \times n^{(m)} \bmod p, \\
& A^{(m+1)} \equiv n_{\ell}^{(m)}+k_{a}^{(m+1)} \bmod 2^{N} .
\end{aligned}
$$

It is vital for the security of the protocol that the updated $k_{b}^{(m+1)}$ and $k_{c}^{(m+1)}$ remain uniformly distributed over $\mathbb{Z}_{p}$ and $\mathbb{Z}_{p} \backslash\{0\}$, respectively. Here is where the updating key, $k_{u}$, comes to play. In addition to inducing a desired independence between message $B^{(m)}$ and the updated $k_{b}^{(m+1)}$, and between message $C^{(m)}$ and the updated $k_{c}^{(m+1)}$, observe that, by Property $2, k_{u}^{(m)}$ will always be uniformly distributed over $\mathbb{Z}_{p}^{*}$ (since the initial $k_{u}^{(0)}$ is drawn uniformly from $\mathbb{Z}_{p}^{*}$ and every generated nonce is a random element of $\mathbb{Z}_{p}^{*}$ ). Therefore, $k_{b}^{(m+1)}$ is uniformly distributed over $\mathbb{Z}_{p}$. However, there is a possibility that $k_{c}^{(m+1)}$ will be equal to zero; which will occur, with negligible probability, when $n^{(m)} \oplus k_{c}^{(m)}$ is congruent to zero modulo $p$. In this case, $n^{(m)} \oplus k_{c}^{(m)}$ in equation (8) is replaced with $n^{(m)} \times k_{c}^{(m)}$. Now, $n^{(m)} \times k_{c}^{(m)}$ is guaranteed not to be congruent to zero (by Property 1), which guarantees that $k_{c}^{(m+1)}$ is not zero. The reason for not starting with $n^{(m)} \times k_{c}^{(m)}$ in the update equation of $k_{c}^{(m+1)}$ is that this is equal to $C^{(m)}$, which will lead to revealing information about the nonce with the observation of multiple consecutive protocol runs. With the update procedure described above, $n^{(m)} \times k_{c}^{(m)}$ will be used for updating $k_{c}^{(m+1)}$ with negligible probability, and even when it is used, the adversary can never know that it is being used. Therefore, without loss of generality, we will assume for the rest of the paper that equation (8) always results in a $k_{c}^{(m+1)}$ that is uniformly distributed over $\mathbb{Z}_{p} \backslash\{0\}$.

\section{Security Analysis}

Before we show the security of our UCS-RFID, we will first prove our claims that, under our adversarial model, the integrity of the delivered nonce, $n$, can be verified using a single modular multiplication, and show that the random nonce is delivered to tags in an unconditionally secure manner.

\subsection{Integrity of the Delivered Nonce}

In this section, we will show how the integrity of the nonce, $n$, is preserved without resorting to computationally secure cryptographic primitives. The integrity of the delivered nonce in our UCS-RFID is accomplished in a novel way, by taking advantage of the properties of the integer field $\mathbb{Z}_{p}$, with only a single multiplication operation.

There are two cases to consider: modifying message $B$ alone and modifying both $B$ and $C$ in order to make the tag authenticate a false nonce. Modifying message $C$ 
alone, since its main purpose is to authenticate the received nonce, does not lead to the extraction of a modified nonce.

Lemma 3 Given that $k_{c}$ is uniformly distributed over $\mathbb{Z}_{p} \backslash\{0\}$, the probability of accepting a modified nonce by a valid tag is at most $1 /(p-1)$.

Proof: Assume that message $B$ has been modified to $B^{\prime}$. This modification will lead to the extraction of a nonce, $n^{\prime}$, different than the authentic $n$ generated by the reader; that is, $n^{\prime} \equiv B^{\prime}-k_{b} \bmod p$. Message $C$, however, is used to verify the integrity of the extracted $n^{\prime}$. Let $n^{\prime} \equiv n+\epsilon \bmod p$; for some $\epsilon \in \mathbb{Z}_{p} \backslash\{0\}$. To be accepted by the tag, $n^{\prime}$ must satisfy the integrity check of equation (4). That is,

$$
n^{\prime} \times k_{c} \equiv(n+\epsilon) \times k_{c} \equiv\left(n \times k_{c}\right)+\left(\epsilon \times k_{c}\right) \stackrel{?}{\equiv} C \equiv n \times k_{c} \bmod p .
$$

Clearly, the congruence in equation (12) will be satisfied only if $\epsilon \times k_{c} \equiv 0 \bmod p$. However, since $k_{c}$ is a nonzero element by design, and $\epsilon \not \equiv 0$ (since $\epsilon \equiv 0$ implies that $n^{\prime} \equiv n \bmod p$ ), Property 1 guarantees that $\epsilon \times k_{c} \not \equiv 0 \bmod p$. Therefore, the congruence of equation (12) can never be satisfied, and any modification of message $B$ alone will be detected with probability one.

The second case to consider here is when both messages $B$ and $C$ are corrupted simultaneously. Assume that message $B$ has been modified so that the extracted nonce becomes $n^{\prime} \equiv n+\epsilon \bmod p$; for some $\epsilon \in \mathbb{Z}_{p} \backslash\{0\}$. Also, assume that message $C$ has been modified to $C^{\prime} \equiv C+\delta \bmod p$, for some $\delta \in \mathbb{Z}_{p} \backslash\{0\}$. The integrity of the extracted $n^{\prime}$ is verified using the received $C^{\prime}$ as follows:

$C+\delta \equiv C^{\prime} \stackrel{?}{\equiv} n^{\prime} \times k_{c} \equiv(n+\epsilon) \times k_{c} \equiv\left(n \times k_{c}\right)+\left(\epsilon \times k_{c}\right) \equiv C+\left(\epsilon \times k_{c}\right) \quad \bmod p$

Equivalently, the false $n^{\prime}$ is accepted only if $\delta \equiv \epsilon \times k_{c}$. Since $k_{c}$ is unknown to the adversary, for any fixed $\delta$, by Property 2, there exists a unique $\epsilon \in \mathbb{Z}_{p} \backslash\{0\}$ that satisfies (13). Therefore, the probability of modifying both $B$ and $C$ in a way undetected by the tag is at most $1 /(p-1)$ (equivalently, guessing the value of $k_{c}$ ).

\subsection{Secrecy of the Delivered Nonce}

Before we show that the nonce is delivered to the tag in an unconditionally secure manner, we need the following lemma.

Lemma 4 Given that the preloaded subkeys $k_{a}^{(0)}, k_{b}^{(0)}, k_{c}^{(0)}$, and $k_{d}^{(0)}$ are mutually independent, the subkeys $k_{a}^{(m)}, k_{b}^{(m)}, k_{c}^{(m)}$, and $k_{d}^{(m)}$ at the $m^{\text {th }}$ protocol run are mutually independent, for any $m \in \mathbb{N}$.

Proof: Let $\boldsymbol{k}_{a}^{(m)}, \boldsymbol{k}_{b}^{(m)}, \boldsymbol{k}_{c}^{(m)}$, and $\boldsymbol{k}_{d}^{(m)}$ be the random variables representing the subkeys involved in the generation of the messages exchanged between an authorized RFID pair during the $m^{t h}$ protocol run of our UCS-RFID. Then, for any $k_{a}^{(1)}, k_{b}^{(1)}, k_{c}^{(1)}$, and $k_{d}^{(1)}$ 


$$
\begin{aligned}
\operatorname{Pr}\left(\boldsymbol{k}_{\boldsymbol{a}}^{(\mathbf{1})}=k_{a}^{(1)}, \boldsymbol{k}_{\boldsymbol{b}}^{(\mathbf{1})}=k_{b}^{(1)}, \boldsymbol{k}_{\boldsymbol{c}}^{(\mathbf{1})}=k_{c}^{(1)}, \boldsymbol{k}_{\boldsymbol{d}}^{(\mathbf{1})}=k_{d}^{(1)}\right) & \cdot \operatorname{Pr}\left(\boldsymbol{n}=n, \boldsymbol{k}_{\boldsymbol{u}}=k_{u}\right) \\
= & \sum_{n, k_{u}} \operatorname{Pr}\left(\boldsymbol{k}_{\boldsymbol{a}}^{(\mathbf{1})}=k_{a}^{(1)}, \boldsymbol{k}_{\boldsymbol{b}}^{(\mathbf{1})}=k_{b}^{(1)}, \boldsymbol{k}_{\boldsymbol{c}}^{(\mathbf{1})}=k_{c}^{(1)}, \boldsymbol{k}_{\boldsymbol{d}}^{(\mathbf{1})}=k_{d}^{(1)} \mid \boldsymbol{n}=n, \boldsymbol{k}_{\boldsymbol{u}}=k_{u}\right) \\
= & \sum_{n, k_{u}} \operatorname{Pr}\left(\boldsymbol{k}_{\boldsymbol{a}}^{(\mathbf{0})}=k_{a}^{(1)} \oplus n_{r}, \boldsymbol{k}_{\boldsymbol{b}}^{(\mathbf{0})}=\left(k_{b}^{(1)}-k_{u}^{(0)}\right) \oplus n, \boldsymbol{k}_{\boldsymbol{c}}^{(\mathbf{0})}=\left(k_{c}^{(1)} \times k_{u}^{(0)}\right)^{-1}\right) \oplus n \\
= & \sum_{n, k_{u}} \operatorname{Pr}\left(\boldsymbol{k}_{\boldsymbol{a}}^{(\mathbf{0})}=k_{a}^{(1)} \oplus n_{r}\right) \cdot \operatorname{Pr}\left(\boldsymbol{k}_{\boldsymbol{b}}^{(\mathbf{0})}=\left(k_{b}^{(1)}-k_{u}^{(0)}\right) \oplus n\right) \\
& \cdot \operatorname{Pr}\left(\boldsymbol{k}_{\boldsymbol{c}}^{(\mathbf{0})}=\left(k_{c}^{(1)} \times k_{u}^{(0)}-1\right) \oplus n\right) \cdot \operatorname{Pr}\left(\boldsymbol{k}_{\boldsymbol{d}}^{(\mathbf{0})}=k_{d}^{(1)} \oplus n_{r}\right) \cdot \operatorname{Pr}\left(\boldsymbol{n}=n, \boldsymbol{k}_{\boldsymbol{u}}=k_{u}\right) \\
= & \sum_{n, k_{u}} \frac{1}{2^{N}} \cdot \frac{1}{p} \cdot \frac{1}{p-1} \cdot \frac{1}{2^{N}} \cdot \operatorname{Pr}\left(\boldsymbol{n}=n, \boldsymbol{k}_{\boldsymbol{u}}=k_{u}\right) \\
= & \operatorname{Pr}\left(\boldsymbol{k}_{\boldsymbol{a}}^{(\mathbf{1})}=k_{a}^{(1)}\right) \cdot \operatorname{Pr}\left(\boldsymbol{k}_{\boldsymbol{b}}^{(\mathbf{1})}=k_{b}^{(1)}\right) \cdot \operatorname{Pr}\left(\boldsymbol{k}_{\boldsymbol{c}}^{(\mathbf{1})}=k_{c}^{(1)}\right) \cdot \operatorname{Pr}\left(\boldsymbol{k}_{\boldsymbol{d}}^{(\mathbf{1})}=k_{d}^{(1)}\right) .
\end{aligned}
$$

Equations (16) and (17) hold due to the independence and the uniform distribution of the initial subkeys $\left(\boldsymbol{k}_{a}^{(0)}, \boldsymbol{k}_{b}^{(0)}, \boldsymbol{k}_{c}^{(0)}, \boldsymbol{k}_{d}^{(0)}\right)$, respectively; while equation (18) holds due to the uniform distribution of the updated subkeys $\left(k_{a}^{(1)}, k_{b}^{(1)}, k_{c}^{(1)}, k_{d}^{(1)}\right)$. The existence of $k_{u}^{(0)^{-1}}$, the multiplicative inverse of $k_{u}^{(0)}$ in $\mathbb{Z}_{p}$, is a direct consequence of the fact that $k_{u}^{(0)} \in \mathbb{Z}_{p}^{*}$. The proof of the lemma follows by induction.

Lemma 5 At each instance of the protocol, the random nonce generated by the authorized reader in an instance of our UCS-RFID protocol is delivered to the tag in a perfectly secret manner.

Proof: Fix $k_{b}, k_{c}$, and let $\boldsymbol{n}$ be uniformly distributed over $\mathbb{Z}_{p} \backslash\{0\}$. (Recall that, by Lemma $4, k_{b}$ and $\boldsymbol{k}_{c}$ are statistically independent in every protocol run; so, the superscript will be dropped for ease of notation.) Then the resulting $\boldsymbol{B}$ and $\boldsymbol{C}$ will be uniformly distributed over $\mathbb{Z}_{p}$ and $\mathbb{Z}_{p} \backslash\{0\}$ (by Property 2), respectively. Consequently, for any arbitrary $b \in \mathbb{Z}_{p}$ and $c \in \mathbb{Z}_{p} \backslash\{0\}$, the probability of $\boldsymbol{B}$ and $\boldsymbol{C}$ taking these specific values are $\operatorname{Pr}(\boldsymbol{B}=b)=1 / p$ and $\operatorname{Pr}(\boldsymbol{C}=c)=1 /(p-1)$.

Now, given a specific value of the random nonce $\boldsymbol{n}=n$, the probability that $\boldsymbol{B}$ takes a value $b$ is

$$
\operatorname{Pr}(\boldsymbol{B}=b \mid \boldsymbol{n}=n)=\operatorname{Pr}\left(\boldsymbol{k}_{\boldsymbol{b}}=b-n\right)=1 / p .
$$

Similarly, given a specific value of the random nonce $\boldsymbol{n}=n$, the probability that $\boldsymbol{C}$ takes a value $c$ is

$$
\operatorname{Pr}(\boldsymbol{C}=c \mid \boldsymbol{n}=n)=\operatorname{Pr}\left(\boldsymbol{k}_{\boldsymbol{c}}=c \times n^{-1}\right)=1 /(p-1) .
$$


Equations (19) and (20) hold since, by design, $\boldsymbol{k}_{\boldsymbol{b}}$ and $\boldsymbol{k}_{\boldsymbol{c}}$ are uniformly distributed over $\mathbb{Z}_{p}$ and $\mathbb{Z}_{p} \backslash\{0\}$, respectively. The existence of $n^{-1}$ is a direct consequence of the fact that $n \in \mathbb{Z}_{p}^{*}$.

Therefore, for any nonce $n$ and any values of $b$ and $c$, Bayes' theorem [55] can be used to show that $\operatorname{Pr}(\boldsymbol{n}=n \mid \boldsymbol{B}=b)=\operatorname{Pr}(\boldsymbol{n}=n)=\operatorname{Pr}(\boldsymbol{n}=n \mid \boldsymbol{C}=c)$. That is, the a priori probabilities that the random nonce is $n$ are the same as the a posteriori probabilities that the random nonce is $n$ given the corresponding $B$ and $C$. Hence, both $B$ and $C$ "individually" provided perfect secrecy. However, since they are both functions of the same variable, there might be information leakage about $n$ revealed by the combination of $B$ and $C$. One way of measuring how much information is learned by the observation of two quantities is the notion of mutual information. Consider an arbitrary $b \in \mathbb{Z}_{p}$ and arbitrary $c, n \in \mathbb{Z}_{p}^{*}$. Then, for independent $\boldsymbol{k}_{\boldsymbol{b}}$ and $\boldsymbol{k}_{\boldsymbol{c}}$ uniformly distributed over $\mathbb{Z}_{p}$ and $\mathbb{Z}_{p}^{*}$, respectively, we get:

$$
\begin{aligned}
\operatorname{Pr}(\boldsymbol{B}=b, \boldsymbol{C}=c) & =\sum_{n} \operatorname{Pr}(\boldsymbol{B}=b, \boldsymbol{C}=c \mid \boldsymbol{n}=n) \operatorname{Pr}(\boldsymbol{n}=n) \\
& =\sum_{n} \operatorname{Pr}\left(\boldsymbol{k}_{\boldsymbol{b}}=b-n, \boldsymbol{k}_{\boldsymbol{c}}=c \times n^{-1}\right) \operatorname{Pr}(\boldsymbol{n}=n) \\
& =\sum_{n} \operatorname{Pr}\left(\boldsymbol{k}_{\boldsymbol{b}}=b-n\right) \operatorname{Pr}\left(\boldsymbol{k}_{\boldsymbol{c}}=c \times n^{-1}\right) \operatorname{Pr}(\boldsymbol{n}=n) \\
& =\sum_{n} \frac{1}{p} \cdot \frac{1}{p-1} \cdot \operatorname{Pr}(\boldsymbol{n}=n) \\
& =\operatorname{Pr}(\boldsymbol{B}=b) \cdot \operatorname{Pr}(\boldsymbol{C}=c)
\end{aligned}
$$

Equation (23) holds by the independence of $\boldsymbol{k}_{\boldsymbol{b}}$ and $\boldsymbol{k}_{\boldsymbol{c}}$, while equations (24) and (25) hold by the uniform distribution of $\boldsymbol{k}_{\boldsymbol{b}}, \boldsymbol{k}_{\boldsymbol{c}}, \boldsymbol{B}$, and $\boldsymbol{C}$. Consequently, $\boldsymbol{B}$ and $\boldsymbol{C}$ are independent and, thus, their mutual information is zero [56]. In other words, observing both messages $B$ and $C$ gives no extra information about $n$ than what they give individually.

Remark 1 Lemma 5 does not hold for an adversary who has observed multiple consecutive protocol runs between authorized reader-tag pairs. Consider observing three consecutive $B$ messages, say $B^{(0)}, B^{(1)}, B^{(2)}$. The fundamental problem is that only $k_{b}^{(0)} \in \mathbb{Z}_{p}$ and $k_{u}^{(0)} \in \mathbb{Z}_{p}^{*}$ are involved in the update equation of the subkey $k_{b}$. Therefore, out of the total $(p-1)^{3}$ possible sequences of $\left\{n^{(0)}, n^{(1)}, n^{(2)}\right\}$, to an adversary who has observed $\left\{B^{(0)}, B^{(1)}, B^{(2)}\right\}$, there are only $p(p-1)$ possible $\left\{n^{(0)}, n^{(1)}, n^{(2)}\right\}$ sequences that could have generated the observed $B$ 's. A violation to the definition of perfect secrecy. Obviously, one can include more variables in the update equation of $k_{b}$ but that will only increase the number of consecutive protocol runs an adversary is allowed to observe to a certain number.

However, breaking perfect secrecy does not imply breaking the system. In what follows, we provide an example to further illustrate the remark; then, we give detailed probabilistic analysis to show that the system can still provide unconditional security given some practical assumptions about the RFID system. 
Example 1 This example illustrates the effect of observing consecutive protocol runs between authorized reader-tag pairs. For simplicity, assume that the used prime number is $p=7$. Assume further that the initial keys $k_{b}^{(0)}=2$ and $k_{u}^{(0)}=5$ are preloaded into the tag. Consider the first three protocol runs as follows.

First run: let the generated nonce be $n_{0}=1$. Then by equation (2) the adversary can observe $B_{0}=3$ broadcasted by the reader. The tag and the reader will then update the keys according to equations (7) and (10) to $k_{b}^{(1)}=1$ and $k_{u}^{(1)}=5$.

Second run: let the generated nonce be $n_{1}=6$. Then by equation (2) the adversary can observe $B_{1}=0$. The tag and the reader will then update the keys according to equations (7) and (10) to $k_{b}^{(2)}=5$ and $k_{u}^{(2)}=2$.

Third run: let the generated nonce be $n_{2}=2$. Then the adversary can observe $B_{2}=0$. Now, with some algebra the adversary can construct the following system of equations:

$$
\begin{aligned}
& B_{0}=k_{b}^{(0)}+n_{0}, \\
& B_{1}=k_{u}^{(0)}+\left(n_{0} \oplus k_{b}^{(0)}\right)+n_{1}, \\
& B_{2}=\left(k_{u}^{(0)} \times n_{0}\right)+\left(n_{1} \oplus\left(k_{u}^{(0)}+\left(n_{0} \oplus k_{b}^{(0)}\right)\right)\right)+n_{2} .
\end{aligned}
$$

Consider now the sequence $\left\{n_{0}=1, n_{1}=1, n_{2}=1\right\}$. Given the observed $B$ 's, by checking equations (26), (27), and (28), one can see that the sequence $\left\{n_{0}=1, n_{1}=\right.$ $\left.1, n_{2}=1\right\}$ cannot satisfy the three equations simultaneously. Moreover, by checking all possible $6 \times 6 \times 6$ sequences, one can find that only $7 \times 6$ of them can satisfy all three equations simultaneously. The fundamental problem is that only $k_{b}^{(0)} \in\{0,1,2,3,4,5,6\}$ and $k_{u}^{(0)} \in\{1,2,3,4,5,6\}$ are involved in the three equations.

Observe, however, that this does not imply anything more than that the sequence $\left\{n_{0}=1, n_{1}=1, n_{2}=1\right\}$ cannot generate the observed $B$ 's. That is, it does not imply that $n_{0} \neq 1$, nor that $n_{1} \neq 1$, nor that $n_{2} \neq 1$. In other words, individually, any one of the $n$ 's can be equal to one (indeed, $n_{0}=1$ in the above example).

Therefore, for the adversary to obtain meaningful information, she must know the exact value of at least one of the nonces (so that possible values of other nonces can be eliminated). This can only occur if for at least one nonce $n_{i}$, only one value in $\mathbb{Z}_{p}^{*}$ is possible. That is, all the possible values $n_{i}$ is allowed to take can be eliminated, except for exactly one value.

We will now provide probabilistic analysis of the number of consecutive protocol runs an adversary must observe in order to learn the value of at least one of the transmitted nonces.

By the randomness nature of the generated nonces, the total number of possible sequences is uniformly distributed over the nonces. That is, given there are $p(p-1)$ possible sequences, if the adversary has observed $m$ consecutive protocol runs, each of the $m$ nonces is expected to have $\sqrt[m]{p(p-1)}$ possible values. Therefore, for $m$ consecutive protocol runs, the total number of possible values distributed over the $m$ nonces is $m \sqrt[m]{p(p-1)}$ 

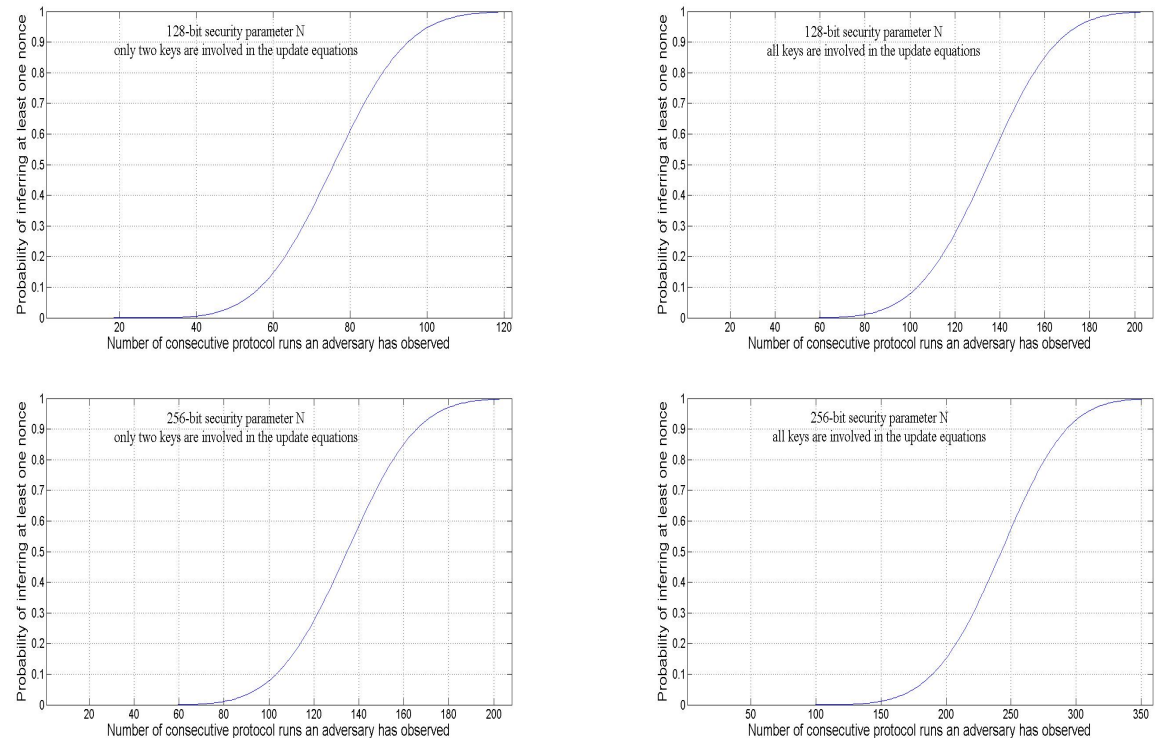

Figure 3. The probability of exposing at least one nonce as a function of the number of consecutive protocol runs observed by the adversary, for different size of security parameter and different number of parameters involved in the update equation.

To give a lower bound on the number of consecutive protocol runs an adversary must observe in order to infer at least one nonce with a certain probability, we use the well-known "balls in bins without capacity" problem in probability theory. Given $r$ balls thrown uniformly at random at $m$ bins, the probability that at least one bin remains empty is given by [57]:

$$
\operatorname{Pr}(\text { at least one bin remains empty })=\frac{\left(\begin{array}{c}
r-1 \\
m-1
\end{array}\right)}{\left(\begin{array}{c}
m+r-1 \\
m-1
\end{array}\right)} \text {. }
$$

Given that each nonce will take at least one value, the problem reduces to distributing $(m \sqrt[m]{p(p-1)}-m)$ values uniformly at random at $m$ nonces and finding the probability that at least one nonce does not receive another possible value. Substituting $r=m \sqrt[m]{p(p-1)}-m$ in equation (29), we plot the results in Figure 3. Each plot shows the number of consecutive protocol runs an adversary must observe in order to infer at least one nonce with a certain probability. In the top left plot, the security parameter $N$ is 128-bit long, with only $k_{b}$ and $k_{u}$ are involved in the update equation of $k_{b}$. The plot in the top right shows the result when all secret keys are involved in the update equation of $k_{b}$. The two bottom plots shows the result when the used security parameter is 256 -bit long.

As can be seen in Figure 3, the number of consecutive protocol runs an adversary has to observe to learn the value of at least one nonce is much higher than the number of protocol runs needed to break perfect secrecy. Depending on how many secret keys are used in the update equations and the length of the security parameter, for an adversary to have a $50 \%$ chance of exposing a secret nonce value, the number of consecutive protocol runs needed to be observed can be as high as 240 complete runs. 
Remark 2 Observe that, unlike general computer communications, that many consecutive protocol runs can be sufficiently high for RFID systems. Consider, for example, an RFID tag used for a pay-at-the-pump application. If the user goes to the same gas station every single time, this implies that for an adversary to extract secret tag information, she must be in a close proximity to the user for about 240 consecutive gas pumping. In the case in which the user goes to different gas stations, this implies that the adversary is following the user everywhere. Both scenarios are highly unlikely to occur in real life applications. In a different example, consider low-cost tags replacing barcodes for identifying grocery items. In such applications, that many authorized protocol runs are unlikely to occur during the entire life time of a low-cost tag. The following corollary is a direct consequence of this remark.

Corollary 1 In order to expose secret tag information, the adversary must observe a sufficiently high number of honest protocol runs between authorized reader-tag pairs.

This implies that adversaries, regardless of their computational power, must rely on authorized reader-tag interactions to have a chance of inferring secret information.

Assumption 1 For the rest of the paper, we will adopt the assumption that observing enough protocol runs to expose the value of a nonce is impractical in low-cost RFID systems.

\subsection{Security of Mutual Authentication}

Before we can state our main theorem regarding the security of mutual authentication in our protocol, we need two more lemmas.

Lemma 6 Under Assumptions 1, given that the reader generates random nonces, no information about the secret key, $K$, is revealed by observing protocol runs of the proposed protocol.

Proof: We start with the basic assumption that the key is loaded to the tag secretly; that is $k_{a}^{(0)}, k_{b}^{(0)}, k_{c}^{(0)}$, and $k_{d}^{(0)}$ are secret. By Lemma 5 , messages $B^{(0)}$ and $C^{(0)}$ provide perfect secrecy. That is, no information about the nonce, $n^{(0)}$, nor the keys, $k_{b}^{(0)}$ and $k_{c}^{(0)}$, will be leaked by $B^{(0)}$ and $C^{(0)}$. Now, $n^{(0)}$ will be used to generate $D^{(0)}=n_{\ell}^{(0)} \oplus k_{d}^{(0)}$ and $A^{(1)}=n_{\ell}^{(0)}+\left(n_{r}^{(0)} \oplus k_{a}^{(0)}\right)$. Since $n^{(0)}$ is delivered in a perfectly secret manner, and $k_{d}^{(0)}$ and $k_{a}^{(0)}$ are secret, no information will be revealed by the observation of $D^{(0)}$ and $A^{(1)}$. (The proof is very similar to the proof of Lemma 5 ; it is based on the fact that $k_{a}^{(0)}$ and $k_{d}^{(0)}$ are random and independent.)

So far, no secret information about the initial key $K^{(0)}$ nor the nonce $n^{(0)}$ has been revealed. Therefore, there is no information leakage about the updated subkeys $k_{a}^{(1)}=$ $n_{r}^{(0)} \oplus k_{a}^{(0)}, k_{b}^{(1)}=k_{u}^{(0)}+\left(n^{(0)} \oplus k_{b}^{(0)}\right), k_{c}^{(1)}=k_{u}^{(0)} \times\left(n^{(0)} \oplus k_{c}^{(0)}\right)$, and $k_{d}^{(1)}=n_{r}^{(0)} \oplus k_{d}^{(0)}$. Given that the keys are updated to remain independent and to have the same distribution as the outdated keys, and that $n^{(1)}$ is random and independent from the previous nonce and from the secret keys, the proof follows by induction (given Assumption 1).

Lemma 7 Under Assumption 1, an adversary making $q_{q}$ Query oracles, $q_{s}$ Send oracles will succeed with probability at most 


$$
\max \left\{\frac{q_{q}}{p-1}, \frac{q_{s}}{2^{N}}\right\}
$$

Proof: By Lemma 6 and Corollary 1, calling the Execute oracle a practical number of consecutive times is of no help to the adversary, since no information is leaked by observing messages exchanged between authorized RFID pairs.

On the other hand, an adversary calling the Query oracle will receive $A$ as the tag's response. Depending on the adversary's response, the tag will respond with message $D$ with probability $1 /(p-1)$ (the probability of successful forgery by Lemma 3), or abort the protocol with probability $(p-2) /(p-1)$. If the tag does respond, the protocol is considered broken. However, upon unsuccessful forgery, the tag will abort, and responds to the next Query with the same identifier, $A$. Therefore, no information about the tag's secret key is revealed by multiple Query calls.

Finally, an adversary calling the Send oracle to impersonate a valid tag will be successful with probability at most $1 / 2^{N}$. This is due to the fact that $A$ might or might not be a valid tag identifier. If it is not, the reader will abort the protocol. Assume, however, that $A$ is a valid identifier (the adversary can obtain a valid one by interrogating a tag in the system). An authorized reader, responding with $B$ and $C$, will accept $D$ if and only if $D=n_{\ell} \oplus k_{d}$. To extract the correct $n_{\ell}$, however, the adversary must know $k_{b}$ or $k_{c}$, which are kept secret by Lemma 6 . Moreover, $k_{d}$ is unknown to the adversary (also by Lemma 6). Hence, the adversary's probability of success is $1 / 2^{N}$, and the lemma follows.

Given that $p$ is a $2 N$-bit prime integer, the adversary's probability of falsely authenticating herself to a valid tag is at most $1 / 2^{2 N-1}$, and the adversary's probability of authenticating herself to a valid reader is $1 / 2^{N}$. That is, the probability of mutual authentication when the protocol is not honest is negligible in the security parameter $N$. We can now state our main theorem.

Theorem 1 Under Assumption 1, the proposed UCS-RFID is a secure mutual authentication protocol for RFID systems.

Proof: Lemma 6 implies that the first condition of Definition 2 is satisfied. The second condition of Definition 2 can be easily verified; it merely means that if the messages exchanged between legitimate RFID pairs are relayed faithfully to one another, mutual authentication is achieved. The third condition of Definition 2 is shown to be satisfied in Lemma 7. Thus, all three conditions of Definition 2 are satisfied.

\section{The Block Oracle}

Given the adversarial model of Section 3.2, the protocol described in Section 4 is a secure mutual authentication for RFID systems. However, there are other possible attacks that can be launched if the adversary has the ability to block some of the exchanged messages. In this section, we introduce the Block oracle to model the adversary's ability to block exchanged messages and discuss the possible attacks that can be launched using the Block oracle. 


\subsection{Desynchronization Attacks}

As in stateful protocols, the update procedure is critical for the security and the correctness of the protocol. Without a valid identifier and a valid set of keys, tags cannot be identified successfully in the proposed protocol. Consequently, not only the secrecy and authenticity of tags parameters must be preserved, but also tags' states must be synchronized with the database. That is, if the parameters of a certain tag in the database are different than the parameters stored at the tag itself, the tag cannot be identified successfully by authorized readers. Therefore, it is important to show that tags in the proposed system are secured against possible desynchronization attacks.

From Theorem 1, an adversary cannot cause a desynchronization between tags and the database with a non-negligible probability by authenticating herself to the tag or the reader. The protocol as described in Section 4, however, allows an adversary to mount a desynchronization attack by blocking some messages exchanged between authorized reader-tag pairs. In order to formalize such attacks, we add the Block oracle to the oracles defined in Section 3.2

- Block $(\cdot): \mathcal{A}$ blocks any part of the protocol. This query models the adversary's ability to block exchanged messages in order to launch desynchronization attacks.

In what follows, we investigate the effect of blocking different messages in a protocol run and then give an extension to the protocol description of Section 4.

1. Block("Hello"): An adversary blocking the first protocol message, the "Hello" message from the reader to the tag, will cause the tag not to respond. That is, neither the tag nor the database will update the tag's state. Therefore, no desynchronization will occur by blocking the first protocol message.

2. $\operatorname{Block}(A)$ : Consider an adversary blocking the second message containing the tag's identifier $A$. Again, neither the tag nor the database will update the tag's parameters and no desynchronization will occur. An adversary replaying message $A$ to the reader will cause no harm either. To see this, recall that the adversary does not know $k_{b}, k_{c}$ nor $k_{d}$, hence, she cannot extract the correct $n$ and generate a valid $D$ with a non-negligible probability.

3. $\operatorname{Block}(B, C)$ : Consider an adversary blocking messages $B$ and $C$, and replaying them to the tag. Of course, the adversary will be authenticated. However, this is considered as faithfully relaying messages, which does not affect the honesty of the protocol. This makes sense, because the tag will respond with a message $D$ which does not reveal extra information about the tag that has not been revealed by $A$.

4. $\operatorname{Block}(D)$ : Consider an adversary blocking message $D$ sent to the reader. The reader will assume that the tag has not updated its parameters while, in fact, it has. Consequently, the secret keys at the tag's side will be different than the secret keys stored at the database, causing a possible desynchronization between the tag and the reader. A solution to this problem is that the reader updates the parameters even if it does not receive message $D$ from the tag. The reader, however, must store both the updated and the outdated parameter values at the database to count for the possible scenario that the tag has not updated its parameters (more details in Section 7). 


\subsection{Key Exposure}

A more dangerous attack can be launched by blocking messages $B$ and $C$ sent to the tag, or message $D$ sent to the reader, if the same keys, with different nonces are used in the next protocol run. To see this, assume that $B^{(1)} \equiv n^{(1)}+k_{b}^{(1)} \bmod p$ and $C^{(1)} \equiv$ $n^{(1)} \times k_{c}^{(1)} \bmod p$ have been blocked by an active adversary. If the same keys $k_{b}^{(1)}$ and $k_{c}^{(1)}$ are used to generate $B^{(2)} \equiv n^{(2)}+k_{b}^{(1)} \bmod p$ and $C^{(2)} \equiv n^{(2)} \times k_{c}^{(1)} \bmod p$, the difference between the two nonces, $n^{(1)}$ and $n^{(2)}$, is simply the difference between $B^{(1)}$ and $B^{(2)}$. It can be easily seen that:

$$
\begin{aligned}
C^{(2)} & \equiv n^{(2)} \times k_{c}^{(1)} \bmod p \\
& \equiv\left(n^{(1)}+\delta\right) \times k_{c}^{(1)} \bmod p \\
& \equiv\left(n^{(1)} \times k_{c}^{(1)}\right)+\left(\delta \times k_{c}^{(1)}\right) \quad \bmod p \\
& \equiv C^{(1)}+\left(\delta \times k_{c}^{(1)}\right) \quad \bmod p .
\end{aligned}
$$

Hence, with the knowledge that $n^{(2)} \equiv n^{(1)}+\delta \bmod p$, where $\delta \equiv B^{(2)}-B^{(1)}$ $\bmod p$, the value of $k_{c}^{(1)}$ can be easily computed as $k_{c}^{(1)} \equiv\left(C^{(2)}-C^{(1)}\right) \times \delta^{-1} \bmod p$. Thus, we emphasize that whenever the reader receives an outdated identifier, the reader retransmits the same messages $B^{(1)}$ and $C^{(1)}$, as opposed to generating a new nonce and transmitting $B^{(2)}$ and $C^{(2)}$ as above.

The requirement that the reader responds with the same $B$ and $C$ when receiving an outdated $A$, however, introduces a vulnerability to a man-in-the-middle (MITM) attack. Consider an adversary observing messages $A^{(1)}, B^{(1)}, C^{(1)}$, and then intercepting message $D^{(1)}$. The reader will assume that the tag has not updated its parameter. Hence, in the next protocol run, the adversary can impersonate the tag by sending its $A^{(1)}$ and, upon receiving the same $B^{(1)}$ and $C^{(1)}$, she can replay the intercepted $D^{(1)}$, which will be accepted by the reader.

Fortunately, there is an easy fix for this vulnerability. Whenever a valid reader receives an outdated identifier $A^{(1)}$, it responds with the same $B^{(1)}$ and $C^{(1)}$ to avoid key exposure (as discussed above). But the tag does not get authenticated upon the reception of $D^{(1)}$ (to avoid the man-in-the-middle attack described above). The reader continues by carrying out another protocol run with the tag (with updated keys this time), and only if the second authentication run is passed, with updated parameters to generate $A^{(2)}, B^{(2)}, C^{(2)}$, and $D^{(2)}$, the tag is authenticated. Below, we extend the basic description of our protocol to take into account the desynchronization and key exposure attacks described above.

\section{The Complete UCS-RFID Description}

As discussed in the previous section, the basic protocol description of Section 4 is vulnerable to desynchronization and key recovery attacks. We she below an extension the basic protocol description that is secure against the desynchronization and key recovery attacks illustrated in the previous section. 
Step 1. The reader announces its presence by broadcasting a "Hello" message.

Step 2. The tag responds to the "Hello" message by sending its current identifier, $A$.

Step 3. The reader looks up the database for the key $K=\left(k_{a}, k_{b}, k_{c}, k_{d}, k_{u}\right)$ corresponding to the tag's current identifier, $A$. If $A$ is not recognized as a valid identifier, the tag is rejected.

Step 4. There are two possible scenarios if $A$ is identified: 1 . $A$ can be updated (corresponding to the case in which the tag has updated its parameters successfully during its last protocol run) and, 2. $A$ can be outdated (corresponding to the case in which the tag has not updated its parameters during its last protocol run, while the database has).

4.1. If $A$ is updated, the reader generates a $2 N$-bit random nonce, $n$, drawn uniformly at random from the multiplicative group $\mathbb{Z}_{p}^{*}$. The reader also deletes the outdated information of the tag from the database, if existed.

4.2. If $A$ is outdated, the reader uses the same nonce $n$ used for the last protocol run with the tag.

Step 5. With $k_{b}, k_{c}$, and $n$, the reader broadcasts two messages, $B$ and $C$, generated according to the following formulas:

$$
\begin{array}{ll}
B \equiv n+k_{b} & \bmod p, \\
C \equiv n \times k_{c} & \bmod p .
\end{array}
$$

Again, there are two possible scenarios.

5.1. If $A$ was updated, the reader updates the tags parameters. Let $A^{(m)}, k_{i}^{(m)}$, and $n^{(m)}$ denote the identifier $A, k_{i}$, and $n$ used to execute the $m^{\text {th }}$ protocol run; let $n_{r}$ denotes the $N$ least significant bits of $n$. Then, the parameters are updated as follows,

$$
\begin{aligned}
& k_{a}^{(m+1)}=n_{r}^{(m)} \oplus k_{a}^{(m)}, \\
& k_{b}^{(m+1)} \equiv k_{u}^{(m)}+\left(n^{(m)} \oplus k_{b}^{(m)}\right) \bmod p, \\
& k_{c}^{(m+1)} \equiv k_{u}^{(m)} \times\left(n^{(m)} \oplus k_{c}^{(m)}\right) \bmod p, \\
& k_{d}^{(m+1)}=n_{r}^{(m)} \oplus k_{d}^{(m)}, \\
& k_{u}^{(m+1)} \equiv k_{u}^{(m)} \times n^{(m)} \bmod p, \\
& A^{(m+1)} \equiv n_{\ell}^{(m)}+k_{a}^{(m+1)} \bmod 2^{N} .
\end{aligned}
$$

5.2. If $A$ was outdated, the reader does nothing (since the parameters have already been updated during the last protocol run).

Step 6. Upon receiving $B$ and $C$, the tag extracts $n$ from message $B$ and verifies its integrity using message $C$. The reader is authenticated if and only if the following integrity check is satisfied,

$$
\left(B-k_{b}\right) \times k_{c} \equiv C \bmod p .
$$


If the integrity check of equation (43) does not pass, the reader will not be authenticated and the tag will abort the protocol. (This is an example where the tag's identifier used in the next protocol run will be outdated.)

Step 7. If the reader is authenticated, the tag broadcasts message $D$, given by

$$
D=n_{\ell} \oplus k_{d}
$$

where $n_{\ell}$ denotes the $N$ most significant bits of $n$ (the subscript $\ell$ refers to the $\ell$ eft half of $n$ ). The tag then updates its parameters according to equations (37)-(42).

Step 8. Upon receiving $D$, the reader authenticates the tag by verifying that the received $D$ is equal to $n_{\ell} \oplus k_{d}$. If $D$ does not pass the validity check, the tag is rejected. If $D$ passes the validity check, there are two possible scenario.

8.1. If $A$ was updated, the tag is authenticated.

8.2. If $A$ was outdated, the reader goes back to Step 1 to start another protocol run with the tag.

\section{Privacy of RFID Tags}

In this section we analyze the level of privacy achieved by our UCS-RFID according to the Definition 3 of our security model. Consider a tag $T_{a}$ that has been interrogated by the adversary to get its identifier $A_{a}^{(i)}$.

Lemma 8 Under our adversarial model, tags executing our protocol are passively private.

Proof: Assume the tag has accomplished mutual authentication with an authorized reader. Its identifier $A_{a}^{(i)}$ is updated according to equation (11) to $A_{a}^{(i+1)}=n_{\ell}^{(i)}+k_{a}^{(i)}$, where both $n_{\ell}^{(i)}$ and $k_{a}^{(i)}$ are unknown, random strings. That is, according to Theorem 1 , the adversary cannot correctly predict the value of $A_{a}^{(i+1)}$ with a non-negligible probability. Therefore, given the tag, $T_{a}$, with identifier $A_{a}^{(i+1)}$, and another tag, $T_{b}$, with identifier $A_{b}$, the adversary can do no better than a random guess. That is, $\operatorname{Pr}\left(T_{g}=T_{a}\right)=0.5$, and the adversary's advantage of tracking the tag is $\operatorname{Adv}_{\mathcal{A}}=2\left(\operatorname{Pr}\left(T_{g}=T_{a}\right)-0.5\right)=0$. Consequently, tags using our protocol are passively private.

Lemma 9 Under our adversarial model, tags executing our protocol are not actively private.

Proof: With the absence of authorized readers, tags are unable to update their parameters. Hence, an adversary interrogating the same tag multiple times will receive the same response, and the adversary's advantage of recognizing the tag is one. Consequently, tags using our UCS-RFID for mutual authentication are not actively private.

The case of tracking tags by their other responses can be handled similarly. Section 8.1 addresses the problem of active privacy and discuss possible solutions. 


\subsection{Tag Probing Attack}

In the tag probing attack, a rogue reader probes the tag by sending a "Hello" message. The tag challenges the reader by revealing its identifier, $A$, and the reader replies with false authentication information. Given that the authentication fails, the tag will not update its keying information and its identifier. Hence, the tag will respond with the same $A$ on every "Hello" message after every false authentication attempt.

This is a common issue shared by all stateful RFID protocols. The fundamental problem here is that tags are identified via their states, the identifiers in the proposed protocol. To prevent illegal tag tracking, the state must be updated. Since the state in both the tag and the database must be the same, to enable identification, the tag cannot update its identifier in a way unrecognized by valid readers. Consequently, active adversaries interrogating the same tag multiple times, without successfully completing the protocol run, will be able to correlate the tag's responses. Thus, leading to the ability to illegally track RFID tags.

Using the tag probing attack, the adversary can track the tag and the user that carries it, until a successful authentication is performed with an authorized reader. The problem of active privacy, however, is very challenging in RFID protocols based on symmetrickey cryptography (see, e.g., [10,58,59,60,61] for references addressing this issue). Most existing protocols that provide active privacy require readers to perform linear search of all tags in the system in order to identify every single tag response [32]. (Although out of the scope of this paper, the identification process can be performed more efficiently in this protocol since identifiers are broadcasted in clear text.) Given the stringent computational power of tags in this protocol, we discuss the following non-cryptographic techniques to mitigate the tag probing vulnerability.

In [62], Juels et al. introduced the idea of a blocker tag. The blocker tag can simulate many ordinary RFID tags simultaneously to enhance users' privacy. In [63], Floerkemeier et al. proposed the use of a watchdog tag. The watchdog tag enables users to be aware of their tags being interrogated. In [64], Rieback et al. proposed the idea of using an RFID guardian. The RFID guardian is a battery powered device that protects tags from being illegally scanned. In [65], Juels et al. proposed the idea of using an RFID Enhancer Proxy (REP) to enhance the privacy of RFID tags.

\section{Conclusion}

In this paper, a new direction into the problem of authenticating low-cost RFID systems is proposed. The aim of this paper was to investigate the possibilities of unconditional security in the design of RFID protocols. An instance of such protocols was proposed. Under a restriction on the number of consecutive protocol runs an adversary is assumed to observe, the proposed protocol is shown to achieve unconditional secrecy and unconditional integrity. The main goal of this new approach is to design secure RFID protocols with minimum hardware requirements to meet the demand of secure low-cost RFID systems. 


\section{References}

[1] A. Juels and R. Pappu, "Squealing euros: Privacy protection in RFID-enabled banknotes," in Financial Cryptography - FC'03, ser. Lecture Notes in Computer Science, R. N. Wright, Ed., vol. 2742, IFCA. Le Gosier, Guadeloupe, French West Indies: Springer-Verlag, January 2003, pp. 103-121.

[2] P. Golle, M. Jakobsson, A. Juels, and P. Syverson, "Universal re-encryption for mixnets," in The Cryptographers' Track at the RSA Conference - CT-RSA, ser. Lecture Notes in Computer Science, T. Okamoto, Ed., vol. 2964. San Francisco, California, USA: Springer-Verlag, February 2004, pp. $163-178$.

[3] M. Burmester, B. De Medeiros, and R. Motta, "Robust, anonymous RFID authentication with constant key-lookup," in Proceedings of the 2008 ACM symposium on Information, computer and communications security. ACM New York, NY, USA, 2008, pp. 283-291.

[4] B. Liang, Y. Li, C. Ma, T. Li, and R. Deng, "On the Untraceability of Anonymous RFID Authentication Protocol with Constant Key-Lookup," 5th International Conference on Information Systems SecurityICISS'09, pp. 71-85, 2009.

[5] G. Avoine, K. Kalach, and J.-J. Quisquater, "ePassport: Securing international contacts with contactless chips," in Financial Cryptography and Data Security - FC'08, 2008.

[6] T. S. Heydt-Benjamin, D. V. Bailey, K. Fu, A. Juels, and T. O'Hare, "Vulnerabilities in First-Generation RFID-Enabled Credit Cards," Manuscript, 2006.

[7] M. Feldhofer, S. Dominikus, and J. Wolkerstorfer, "Strong authentication for RFID systems using the AES algorithm," in Workshop on Cryptographic Hardware and Embedded Systems - CHES 2004, ser. Lecture Notes in Computer Science, M. Joye and J.-J. Quisquater, Eds., vol. 3156, IACR. Boston, Massachusetts, USA: Springer-Verlag, August 2004, pp. 357-370.

[8] M. Feldhofer, J. Wolkerstorfer, and V. Rijmen, "AES Implementation on a Grain of Sand," IEE Proceedings - Information Security, vol. 152, no. 1, pp. 13-20, October 2005.

[9] S. Dominikus, E. Oswald, and M. Feldhofer, "Symmetric Authentication for RFID Systems in Practice," Handout of the Ecrypt Workshop on RFID and Lightweight Crypto, 2005.

[10] D. Molnar and D. Wagner, "Privacy and Security in Library RFID: Issues, Practices, and Architectures," in Conference on Computer and Communications Security - ACM CCS, 2004.

[11] J. Lee and Y. Yeom, "Efficient RFID Authentication Protocols Based on Pseudorandom Sequence Generators," Cryptology ePrint Archive, Report 2008/343, 2008.

[12] C. Ma, Y. Li, R. Deng, and T. Li, "RFID privacy: Relation Between Two Notions, Minimal Condition, and Efficient Construction," in Proceedings of the 16th ACM conference on Computer and communications security. ACM, 2009, pp. 54-65.

[13] S. Weis, S. Sarma, R. Rivest, and D. Engels, "Security and privacy aspects of low-cost radio frequency identification systems," in International Conference on Security in Pervasive Computing - SPC 2003, ser. Lecture Notes in Computer Science, D. Hutter, G. Müller, W. Stephan, and M. Ullmann, Eds., vol. 2802. Boppard, Germany: Springer-Verlag, March 2003, pp. 454-469.

[14] G. Avoine and P. Oechslin, "A Scalable and Provably Secure Hash Based RFID Protocol," in International Workshop on Pervasive Computing and Communication Security - PerSec 2005, 2005.

[15] A. Bogdanov, G. Leander, C. Paar, A. Poschmann, M. J. Robshaw, and Y. Seurin, "Hash Functions and RFID Tags : Mind The Gap," in Proceedings of the 10th International Workshop Cryptographic Hardware and Embedded Systems, CHES'08, 2008.

[16] J. Bringer, H. Chabanne, and T. Icart, "Improved Privacy of the Tree-Based Hash Protocols Using Physically Unclonable Function,” in Security and Cryptography for Networks-SCN'08, 2008.

[17] M. O’Neill (McLoone), "Low-Cost SHA-1 Hash Function Architecture for RFID Tags," in Workshop on RFID Security - RFIDSec'08, 2008.

[18] A. Juels and S. Weis, "Authenticating pervasive devices with human protocols," in Advances in Cryptology - CRYPTO'05, ser. Lecture Notes in Computer Science, V. Shoup, Ed., vol. 3126, IACR. Santa Barbara, California, USA: Springer-Verlag, August 2005, pp. 293-308.

[19] H. Gilbert, M. Robshaw, and Y. Seurin, "HB\#: Increasing the Security and Efficiency of HB," in $A d-$ vances in Cryptology - EUROCRYPT'08. Springer, 2008.

[20] J. Bringer, H. Chabanne, and D. Emmanuelle, "HB ${ }^{++}$: a Lightweight Authentication Protocol Secure against Some Attacks," in Security, Privacy and Trust in Pervasive and Ubiquitous ComputingSecPerU'06, 2006.

[21] J. Bringer and H. Chabanne, "Trusted-HB: A Low-Cost Version of $\mathrm{HB}^{+}$Secure Against Man-in-theMiddle Attacks," IEEE Transactions on Information Theory, 2008. 
[22] K. Ouafi, R. Overbeck, and S. Vaudenay, "On the Security of HB\# against a Man-in-the-Middle Attack," in Advances in Cryptology - Asiacrypt 2008, 2008.

[23] I. Vajda and L. Buttyán, "Lightweight authentication protocols for low-cost RFID tags," in Second Workshop on Security in Ubiquitous Computing - Ubicomp 2003, Seattle, WA, USA, October 2003.

[24] P. Peris-Lopez, J. C. Hernandez-Castro, J. Estevez-Tapiador, and A. Ribagorda, "LMAP: A real lightweight mutual authentication protocol for low-cost RFID tags," Printed handout of Workshop on RFID Security - RFIDSec 06, Ecrypt, Graz, Austria, July 2006.

[25] - "M2AP: A minimalist mutual-authentication protocol for low-cost RFID tags," in International Conference on Ubiquitous Intelligence and Computing - UICO6, ser. Lecture Notes in Computer Science, vol. 4159. Springer-Verlag, September 2006, pp. 912-923.

[26] T. Li and R. H. Deng, "Vulnerability analysis of EMAP - an efficient RFID mutual authentication protocol," in Second International Conference on Availability, Reliability and Security - AReS 2007, Vienna, Austria, April 2007.

[27] H. Gilbert, M. Robshaw, and H. Sibert, "Active attack against $\mathrm{HB}^{+}$: a provably secure lightweigh authentication protocol," p. 1169, 2005.

[28] B. Alomair, L. Lazos, and R. Poovendran, "Passive attacks on a class of authentication protocols for RFID," International Conference on Information Security and Cryptology - ICISC, vol. 4817, pp. 102 115, November 2007.

[29] B. Alomair and R. Poovendran, "On the Authentication of RFID Systems with Bitwise Operations," New Technologies, Mobility and Security, 2008. NTMS'08., 2008.

[30] G. Avoine, "Privacy issues in RFID banknote protection schemes," in International Conference on Smart Card Research and Advanced Applications - CARDIS, J.-J. Quisquater, P. Paradinas, Y. Deswarte, and A. Abou El Kadam, Eds., IFIP. Toulouse, France: Kluwer Academic Publishers, August 2004, pp. 33-48.

[31] M. Ohkubo, K. Suzuki, and S. Kinoshita, "Cryptographic Approach to "Privacy-Friendly" Tags," in RFID Privacy Workshop, 2003.

[32] G. Avoine, E. Dysli, and P. Oechslin, "Reducing time complexity in RFID systems," Selected Areas in Cryptography-SAC, vol. 3897, pp. 291-306, 2005

[33] H.-Y. Chien, "SASI: A New Ultralightweight RFID Authentication Protocol Providing Strong Authentication and Strong Integrity," IEEE Transactions on Dependable and Secure Computing, 2007.

[34] B. Song and C. J. Mitchell, "RFID Authentication Protocol for Low-cost Tags," in ACM Conference on Wireless Network Security, WiSec'08, 2008.

[35] M. Feldhofer and C. Rechberger, "A Case Against Currently Used Hash Functions in RFID Protocols," in Workshop on RFID Security - RFIDSec'06. Graz, Austria: Ecrypt, July 2006.

[36] T. Lim, T. Li, and Y. Li, "A Security and Performance Evaluation of Hash-Based RFID Protocols," 4th International Conferences on Information Security and Cryptology-Inscrypt'08, pp. 406-424, 2008

[37] N. Hopper and M. Blum, "Secure human identification protocols," in Advances in Cryptology - ASIACRYPT 2001, ser. Lecture Notes in Computer Science, vol. 2248/2001. Springer, 2001.

[38] J. Katz and A. Smith, "Analyzing the HB and HB+ protocols in the "large error" case," Cryptology ePrint Archive, Report 2006/326, IACR, 2006

[39] P. Peris-Lopez, J. C. Hernandez-Castro, J. M. Estevez-Tapiador, and A. Ribagorda, "EMAP: An efficient mutual authentication protocol for low-cost RFID tags." in OTM Federated Conferences and Workshop: IS Workshop - IS'06, ser. Lecture Notes in Computer Science, vol. 4277. Springer-Verlag, November 2006, pp. 352-361.

[40] B. Defend, K. Fu, and A. Juels, "Cryptanalysis of two lightweight RFID authentication schemes," in International Workshop on Pervasive Computing and Communication Security - PerSec 2007, IEEE. New York, USA: IEEE Computer Society Press, March 2007, pp. 211-216.

[41] T. Li and G. Wang, "Security analysis of two ultra-lightweight RFID authentication protocols," in IFIP SEC 2007. Sandton, Gauteng, South Africa: IFIP, May 2007.

[42] G. Avoine, "Adversarial model for radio frequency identification," in IACR E-print report 2005, vol. 49. Citeseer, 2005

[43] J. Ha, S. Moon, J. Zhou, and J. Ha, "A new formal proof model for RFID location privacy," in Proceedings of the 13th European Symposium on Research in Computer Security: Computer Security. Springer, 2008

[44] Y. Li and X. Ding, "Protecting RFID communications in supply chains," in Proceedings of the 2nd ACM symposium on Information, computer and communications security. ACM, 2007. 
[45] S. Sarma, S. Weis, and D. Engels, "RFID systems and security and privacy implications," in Cryptographic Hardware and Embedded Systems - CHES 2002, ser. Lecture Notes in Computer Science, B. Kaliski, c. Kaya ço, and C. Paar, Eds., vol. 2523. Redwood Shores, CA, USA: Springer-Verlag, August 2002, pp. 454-469.

[46] A. Juels, "RFID security and privacy: A research survey," Manuscript, RSA Laboratories, September 2005.

[47] P. Peris-Lopez, J. C. Hernandez-Castro, J. Estevez-Tapiador, and A. Ribagorda, "RFID systems: A survey on security threats and proposed solutions," in 11th IFIP International Conference on Personal Wireless Communications - PWC06, ser. Lecture Notes in Computer Science, vol. 4217. Springer-Verlag, September 2006, pp. 159-170.

[48] M. Lehtonen, T. Staake, F. Michahelles, and E. Fleisch, "From identification to authentication - a review of RFID product authentication techniques," Printed handout of Workshop on RFID Security - RFIDSec 06, Ecrypt, Graz, Austria, July 2006.

[49] T. van Deursen and S. Radomirović, "Attacks on RFID Protocols," Cryptology ePrint Archive, Report 2008/310, IACR, July 2008.

[50] B. Alomair and R. Poovendran, "Securing Low-cost RFID Systems: an Unconditionally Secure Approach," The 2010 RFID Security Workshop-RFIDsec'10 Asia, 2010.

[51] O. Goldreich, Foundations of Cryptography. Cambridge University Press, 2001.

[52] C. Shannon, Communication Theory and Secrecy Systems. Bell Telephone Laboratories, 1949.

[53] D. Stinson, Cryptography: Theory and Practice. CRC Press, 2002.

[54] B. Alomair and R. Poovendran, "Information Theoretically Secure Encryption with Almost Free Authentication," Journal of Universal Computer Science-J.UCS, vol. 15, no. 15, pp. 2937-2956, 2009, http://www.jucs.org/jucs_15_15/information_theoretically_secure_encryption.

[55] J. Gubner, Probability and Random Processes for Electrical and Computer Engineers. Cambridge University Press, 2006.

[56] T. Cover and J. Thomas, Elements of Information Theory. Wiley-Interscience New York, 2006.

[57] W. Feller, An Introduction to Probability Theory and its Applications. Wiley India Pvt. Ltd., 2008.

[58] M. Burmester, B. de Medeiros, and R. Motta, "Anonymous RFID authentication supporting constantcost key-lookup against active adversaries," Journal of Applied Cryptography, vol. 1, no. 2, pp. 79-90, 2008.

[59] J. Wu and D. R. Stinson, "A Highly Scalable RFID Authentication Protocol," in Proceedings of the 14th Austrialasian Conference on Information Security and Privacy - ACISP'09, Brisbane, Australia, July 2009.

[60] J. H. Cheon, J. Hong, and G. Tsudik, "Reducing RFID Reader Load with the Meet-in-the-Middle Strategy," Cryptology ePrint Archive, Report 2009/092, IACR, 2009.

[61] B. Alomair, A. Clark, J. Cuellar, and R. Poovendran, "Scalable RFID Systems: a Privacy-Preserving Protocol with Constant-Time Identification," in the 40th Annual IEEE/IFIP International Conference on Dependable Systems and Networks - DSN'10, IEEE. Chicago, Illinois, USA: IEEE Computer Society, June 2010.

[62] A. Juels, R. Rivest, and M. Szydlo, "The blocker tag: Selective blocking of RFID tags for consumer privacy," in Proceedings of the 10th ACM conference on Computer and communications security. ACM New York, NY, USA, 2003, pp. 103-111.

[63] C. Floerkemeier, R. Schneider, and M. Langheinrich, "Scanning with a Purpose-Supporting the Fair Information Principles in RFID Protocols," 2nd International Symposium on Ubiquitous Computing Systems, Tokyo, Japan, 2004.

[64] M. Rieback, B. Crispo, and A. Tanenbaum, "RFID Guardian: A battery-powered mobile device for RFID privacy management," Proc. 10th Australasian Conf. on Information Security and Privacy (ACISP 2005, vol. 3574, pp. 184-194, 2005.

[65] A. Juels, P. Syverson, and D. Bailey, "High-power proxies for enhancing RFID privacy and utility," Proc. of the 5th Workshop on Privacy Enhancing Technologies, 2005. 